\title{
Autophagy: one more Nobel Prize for yeast
}

\author{
Andreas Zimmermann ${ }^{1}$, Katharina Kainz ${ }^{1}$, Aleksandra Andryushkova ${ }^{1}$, Sebastian Hofer ${ }^{1}$, Frank Madeo ${ }^{1,2, *}$ \\ and Didac Carmona-Gutierrez ${ }^{1, *}$ \\ ${ }^{1}$ Institute of Molecular Biosciences, NAWI Graz, University of Graz, Graz, Austria. \\ ${ }^{2}$ BioTechMed Graz, Graz, Austria. \\ * Corresponding Authors: \\ F. Madeo; E-mail: frank.madeo@uni-graz.at; \\ D. Carmona-Gutierrez; E-mail: carmonag@uni-graz.at
}

\begin{abstract}
The recent announcement of the 2016 Nobel Prize in Physiology or Medicine, awarded to Yoshinori Ohsumi for the discoveries of mechanisms governing autophagy, underscores the importance of intracellular degradation and recycling. At the same time, it further cements yeast, in which this field decisively developed, as a prolific model organism. Here we provide a quick historical overview that mirrors both the importance of autophagy as a conserved and essential process for cellular life and death as well as the crucial role of yeast in its mechanistic characterization.
\end{abstract}

More than half a century after the discovery of bulk digestion of cellular components by lysosomes, termed "autophagy" [1,2], the year 2016 marks the latest milestone in the elucidation of this degradation process: in December, Yoshinori Ohsumi will be officially awarded the 2016 Nobel Prize in Physiology or Medicine "for his discoveries of mechanisms for autophagy". This distinction underscores the broad impact of autophagy research over the last decades. Autophagy, which can target specific proteins, protein aggregates, or even whole organelles (reviewed in [3]), has been shown to be involved in various physiological and pathological processes, such as cancer, neurodegeneration, and ultimately the regulation of organismal lifespan (reviewed in [4]). While today, autophagic mechanisms are studied in a broad spectrum of organisms, one of them deserves distinguished mention for having been pioneering and still being instrumental in clarifying how autophagy is regulated: the budding yeast, Saccharomyces cerevisiae. In this December issue of Microbial Cell, we thus pay tribute to the importance of this catabolic process and to yeast as a means to elucidate it by featuring a review article by Daniel Klionsky [5], one of the researchers, who have deeply coined the field. Of note, after 2001, 2006, 2009, and 2013, this year's Nobel Prize adds to the strikingly high number of awards conceded during the past 15 years to honor work performed in yeast.

In the late 1980s, autophagy research had met a dead end. Hitherto, researchers heavily relied on electron mi- croscopy to identify morphological hallmarks of autophagy, most prominently double-membraned autophagosomes sequestering cellular components and their fusion with lysosomes. However, quantitative assessment of autophagic flux was difficult, because no established marker metabolites or proteins were available. A model organism that allowed screening for autophagic markers as well as the genetic constituents for autophagy, was therefore much needed. In 1992, Y. Ohsumi demonstrated the accumulation of autophagosomes in yeast vacuoles (the analog of mammalian lysosomes) upon mutation of vacuolar proteases [6]. The same year, D. Klionsky identified the cytoplasm-to-vacuole (CVT) pathway in yeast [7], corroborating the suitability of this unicellular organism to investigate lysosomal degradation processes.

Remarkably, autophagy in yeast - like in mammalian cells - seemed to respond to starvation conditions, and defects in autophagy could soon be linked to decreased viability under nutrient scarcity. Making use of this effect, 15 autophagy-defective yeast mutants could be isolated from a genetic screen, leading to the discovery of the socalled autophagy-related genes (ATGs) in 1993 [8]. Only little thereafter, in 1995, Noda et al. introduced vacuolar alkaline phosphatase (ALP) activity as the first quantitative, biochemical assay to monitor autophagic activity in yeast [9]. A few years later, the ubiquitination-like Atg12p and Atg8p conjugation systems could be identified [10-12]. Thus, yeast was being instrumental to explore and delineate the mechanistic frame, in which autophagy operates. The discovery of autophagy induction upon inhibition of the nutrient-responsive TOR kinase by rapamycin in 1998 finally embedded autophagy in the physiological response to starvation and paved the way for pharmacological autophagy activation [13]. Mechanistically, TOR phosphorylates components of the Atg1p-initiation complex (in particular Atg13p) and thereby inhibits autophagy induction [14]. Importantly, mammalian TOR has been implicated in a plethora of diseases (reviewed in [15]). The effective haploid yeast system, which allowed various combinations of gene deletions in an unprecedented manner for autophagy 
research, helped discover further elements of the autophagic machinery, among them the yeast analog of the Beclin-1 complex [16]. This phosphatidylinositol 3-kinase (PI-3K) complex is essential for membrane retrieval and autophagosome formation (reviewed in [17]). Indeed, PI$3 \mathrm{~K}$ inhibitors had widely been used as autophagy inhibitors $[18,19]$.

Importantly, the growing field of bioinformatics soon confirmed the evolutionary conservation of ATGs between yeast and humans, e.g. the Atg5-Atg12 and the Atg8-PE conjugation systems $[20,21]$. This circumstance corroborated the status of yeast as a valid model organism for autophagy research. After the autophagic core machinery had been extensively characterized, yeast was used to identify key players of selective autophagic degradation pathways, such as autophagy of mitochondria (termed "mitophagy"), which is mediated by Atg32p [22] and, for instance, has broad implications in the aging process [2325]. Other selective autophagy routes, including pexophagy (peroxisomes) or ER-phagy (endoplasmic reticulum) were also first characterized at the molecular level in yeast $[26,27]$. Recently, the selective autophagic degradation of specific proteins, such as the fatty acid synthase has moved into the spotlight [28]. In fact, it is tempting to speculate that autophagy not only reshapes cells at the organelle level, but also specifically depletes key regulatory proteins to prime cells for starvation periods. Thereby, pharmacological autophagy inducers such as rapamycin or spermidine, which was also originally identified in yeast [29], might mimic nutrient depletion and thus exert their longevity-promoting effects [30].

Today, the mechanistic framework of autophagy is well characterized, in large parts due to studies performed in yeast. Nevertheless, there are many unanswered questions, including the site of autophagosome formation (reviewed in [31]), the role of non-canonical autophagy, which can bypass proteins of the core autophagic machinery [32], the functional connection of the autophagic process with metabolism [33,34], and the exact interplay between autophagy and programmed cell death pathways (reviewed in [35]). Extrapolating from the rich history of autophagy research in yeast (Figure 1), we can expect more answers to come from our unicellular buddy.

\section{ACKNOWLEDGMENTS}

$\mathrm{FM}$ is grateful to the Austrian Science Fund FWF (Austria) for grants P23490-B20, P 29262, P24381, P 29203, P 27893, 11000 and "SFB Lipotox", as well as to BMWFW and the Karl-Franzens University for grant "Unkonventionelle Forschung" and grant DKplus Metabolic and Cardiovascular Diseases to KK and FM. BMWFW for "Flysleep (80.109/0001 -WF/V/3b/2015)". We also acknowledge support from NAWI Graz.

\section{CONFLICT OF INTEREST}

The authors declare no conflict of interest.

\section{COPYRIGHT}

(C) 2016 Zimmermann et al. This is an open-access article released under the terms of the Creative Commons Attribution (CC BY) license, which allows the unrestricted use, distribution, and reproduction in any medium, provided the original author and source are acknowledged.

Please cite this article as: Andreas Zimmermann, Katharina Kainz, Aleksandra Andryushkova, Sebastian Hofer, Frank Madeo and Didac Carmona-Gutierrez (2016). Autophagy: one more Nobel Prize for yeast. Microbial Cell 3(12): 579-581. doi: 10.15698/mic2016.12.544

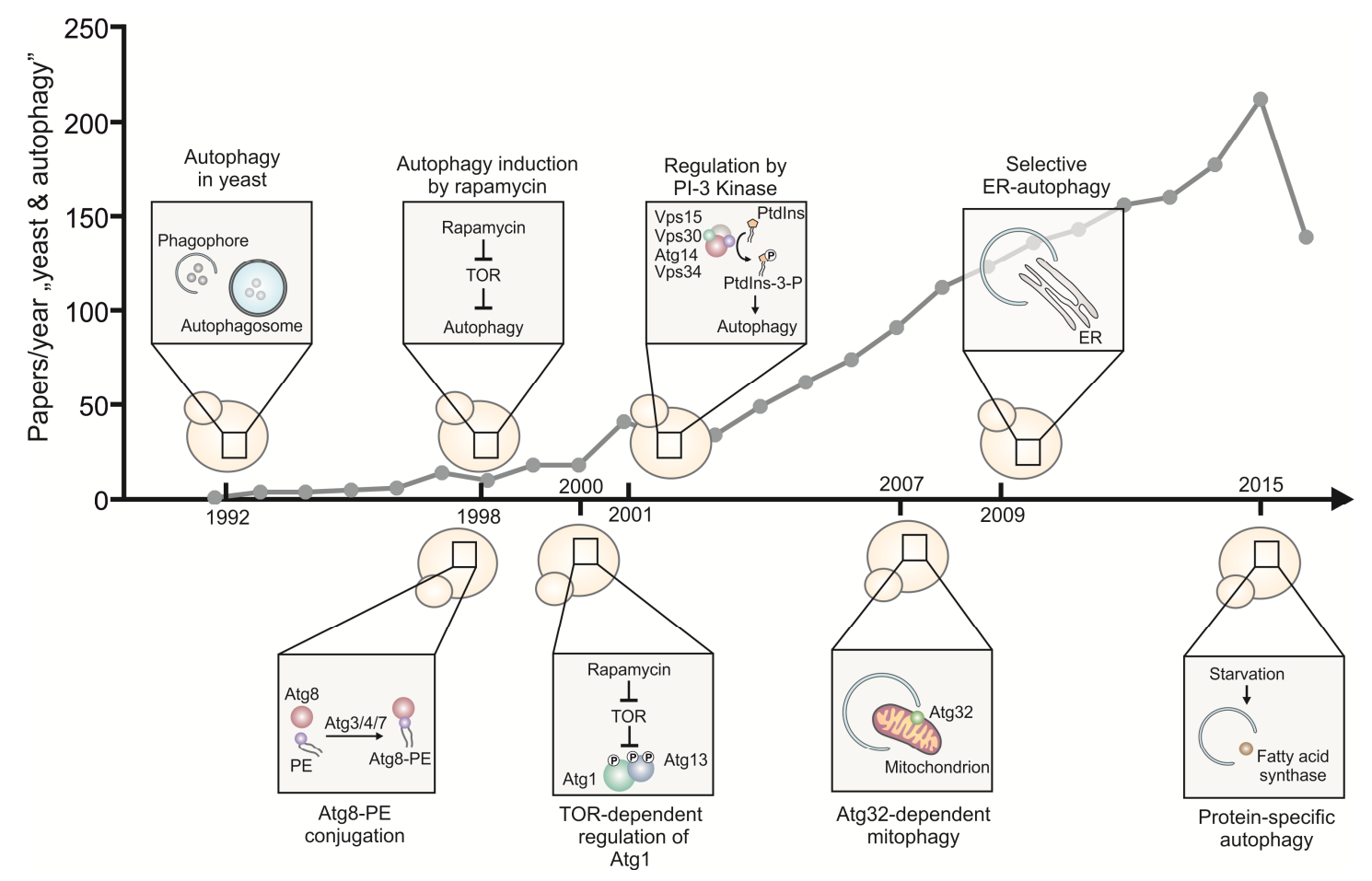

FIGURE 1: A selection of milestones in autophagy research that were first described in yeast and the number of scientific articles per year retrieved in a Pubmed search using the query terms "yeast" and "autophagy". PE, phosphatidylethanolamine. 


\section{REFERENCES}

1. de Duve C (1963) in Ciba Foundation Symposium on Lysosomes. A.V.S. de Reuck and M.P. Cameron, editors. J., and A. Churchill, Ltd., London.

2. Klionsky DJ (2008). Autophagy revisited: a conversation with Christian de Duve. Autophagy 4(6): 740-743.

3. Suzuki K (2013). Selective autophagy in budding yeast. Cell Death Differ 20(1): 43-48.

4. Madeo F, Zimmermann A, Maiuri MC, and Kroemer G (2015). Essential role for autophagy in life span extension. J Clin Invest 125(1): 8593.

5. Zhangyuan Yin, Clarence Pascual and Daniel J. Klionsky (2016). Autophagy: machinery and regulation. Microbial Cell 3(12): 588-596.

6. Takeshige K, Baba M, Tsuboi S, Noda T, and Ohsumi Y (1992). Autophagy in yeast demonstrated with proteinase-deficient mutants and conditions for its induction. J Cell Biol 119(2): 301-311.

7. Klionsky DJ, Cueva R, and Yaver DS (1992). Aminopeptidase I of Saccharomyces cerevisiae is localized to the vacuole independent of the secretory pathway. J Cell Biol 119(2): 287-299.

8. Tsukada M and Ohsumi Y (1993). Isolation and characterization of autophagy-defective mutants of Saccharomyces cerevisiae. FEBS Lett 333(1-2): 169-174.

9. Noda T, Matsuura A, Wada Y, and Ohsumi Y (1995). Novel system for monitoring autophagy in the yeast Saccharomyces cerevisiae. Biochem Biophys Res Commun 210(1): 126-132.

10. Mizushima N, Noda T, Yoshimori T, Tanaka Y, Ishii T, George MD, Klionsky DJ, Ohsumi M, and Ohsumi $Y$ (1998). A protein conjugation system essential for autophagy. Nature 395(6700): 395-398.

11. Lang $T$, Schaeffeler $E$, Bernreuther $D$, Bredschneider $M$, Wolf $D H$ and Thumm $M$ (1998). Aut2p and Aut7p, two novel microtubuleassociated proteins are essential for delivery of autophagic vesicles to the vacuole. EMBO J 17(13): 3597-3607.

12. Kirisako T, Baba M, Ishihara N, Miyazawa K, Ohsumi M, Yoshimori $\mathrm{T}$, Noda T, and Ohsumi Y (1999). Formation process of autophagosome is traced with Apg8/Aut7p in yeast. J Cell Biol 147(2): 435-446.

13. Noda T and Ohsumi Y (1998). Tor, a phosphatidylinositol kinase homologue, controls autophagy in yeast. J Biol Chem 273(7): 39633966.

14. Kamada $Y$, Funakoshi T, Shintani T, Nagano K, Ohsumi M, and Ohsumi Y (2000). Tor-mediated induction of autophagy via an Apg1 protein kinase complex. J Cell Biol 150(6): 1507-1513.

15. Cornu M, Albert V, and Hall MN (2013). mTOR in aging, metabolism, and cancer. Curr Opin Genet Dev 23(1): 53-62.

16. Kihara A, Noda T, Ishihara N, and Ohsumi Y (2001). Two distinct Vps34 phosphatidylinositol 3-kinase complexes function in autophagy and carboxypeptidase $\mathrm{Y}$ sorting in Saccharomyces cerevisiae. J Cell Biol 152(3): 519-530.

17. Backer JM (2008). The regulation and function of Class III PI3Ks: novel roles for Vps34. Biochem J 410(1): 1-17.

18. Blommaart EF, Krause U, Schellens JP, Vreeling-Sindelárová $H$, and Meijer AJ (1997). The phosphatidylinositol 3-kinase inhibitors wortmannin and LY294002 inhibit autophagy in isolated rat hepatocytes. Eur J Biochem 243(1-2): 240-246.

19. Seglen PO and Gordon PB (1982). 3-Methyladenine: specific inhibitor of autophagic/lysosomal protein degradation in isolated rat hepatocytes. Proc Natl Acad Sci U S A 79(6): 1889-1892.
20. Mizushima N, Sugita H, Yoshimori T, and Ohsumi $Y$ (1998). A new protein conjugation system in human. The counterpart of the yeast Apg12p conjugation system essential for autophagy. J Biol Chem 273(51): 33889-33892.

21. Kabeya $\mathrm{Y}$, Mizushima N, Ueno T, Yamamoto A, Kirisako T, Noda T, Kominami E, Ohsumi $Y$, and Yoshimori T (2000). LC3, a mammalian homologue of yeast Apg8p, is localized in autophagosome membranes after processing. EMBO J 19(21): 5720-5728.

22. Kanki T, Wang K, Cao Y, Baba M, and Klionsky DJ (2009). Atg32 is a mitochondrial protein that confers selectivity during mitophagy. Dev Cell 17(1): 98-109.

23. Sampaio-Marques B, Burhans WC, and Ludovico P (2014). Longevity pathways and maintenance of the proteome: the role of autophagy and mitophagy during yeast ageing. Microb Cell 1(4): 118-127.

24. Knorre DA, Sokolov SS, Zyrina AN, and Severin FF (2016). How do yeast sense mitochondrial dysfunction? Microb Cell 3(11): 532-539.

25. Lionaki E, Markaki M, Palikaras K, and Tavernarakis N (2015). Mitochondria, autophagy and age-associated neurodegenerative diseases: New insights into a complex interplay. Biochim Biophys Acta 1847(11): 1412-1423.

26. Hutchins MU, Veenhuis M, and Klionsky DJ (1999). Peroxisome degradation in Saccharomyces cerevisiae is dependent on machinery of macroautophagy and the Cvt pathway. J Cell Sci 112 ( Pt 22): 40794087.

27. Bernales S, Schuck S, and Walter $P$ (2007). ER-phagy: selective autophagy of the endoplasmic reticulum. Autophagy 3(3): 285-287.

28. Shpilka T, Welter E, Borovsky N, Amar N, Shimron F, Peleg Y, and Elazar Z (2015). Fatty acid synthase is preferentially degraded by autophagy upon nitrogen starvation in yeast. Proc Natl Acad Sci U S A 112(5): 1434-1439.

29. Eisenberg T, Knauer H, Schauer A, Büttner S, Ruckenstuhl C, Carmona-Gutierrez D, Ring J, Schroeder S, Magnes C, Antonacci L, Fussi H, Deszcz L, Hartl R, Schraml E, Criollo A, Megalou E, Weiskopf D, Laun P, Heeren G, Breitenbach M, Grubeck-Loebenstein B, Herker E, Fahrenkrog B, Fröhlich K-U, Sinner F, Tavernarakis N, Minois N, Kroemer G, and Madeo F (2009). Induction of autophagy by spermidine promotes longevity. Nat Cell Biol 11(11): 1305-1314.

30. de Cabo R, Carmona-Gutierrez D, Bernier M, Hall MN, and Madeo $F$ (2014). The search for antiaging interventions: from elixirs to fasting regimens. Cell 157(7): 1515-1526.

31. Sanchez-Wandelmer J, Ktistakis NT, and Reggiori F (2015). ERES: sites for autophagosome biogenesis and maturation? J Cell Sci 128(2): 185-192.

32. Scarlatti F, Maffei R, Beau I, Ghidoni R, and Codogno P (2008). Non-canonical autophagy: an exception or an underestimated form of autophagy? Autophagy 4(8): 1083-1085.

33. Schroeder S, Zimmermann A, Carmona-Gutierrez D, Eisenberg T, Ruckenstuhl C, Andryushkova A, Pendl T, Harger A, and Madeo F (2014). Metabolites in aging and autophagy. Microb Cell 1(4): 110114.

34. Galluzzi L, Pietrocola F, Levine B, and Kroemer G (2014). Metabolic Control of Autophagy. Cell 159(6): 1263-1276.

35. Lin L and Baehrecke EH (2015). Autophagy, cell death, and cancer. Mol Cell Oncol 2(3): e985913. 